\title{
Les aveux et dénombrements du Régime français (1723-1745)
}

\section{Jacques Mathieu, Alain Laberge, Renald Lessard et Lina Gouger}

Volume 42, numéro 4, printemps 1989

URI : https://id.erudit.org/iderudit/304737ar

DOI : https://doi.org/10.7202/304737ar

Aller au sommaire du numéro

\section{Éditeur(s)}

Institut d'histoire de l'Amérique française

ISSN

0035-2357 (imprimé)

1492-1383 (numérique)

Découvrir la revue

Citer cet article

Mathieu, J., Laberge, A., Lessard, R. \& Gouger, L. (1989). Les aveux et dénombrements du Régime français (1723-1745). Revue d'histoire de l'Amérique française, 42(4), 545-562. https://doi.org/10.7202/304737ar

\section{Résumé de l'article}

Ce texte présente une analyse critique de la série des aveux et dénombrements du Régime français dont nous voulions vérifier la validité, la représentativité et la fiabilité. Ces documents décrivent, terre par terre, l'implantation de la population dans la vallée laurentienne après le premier quart du XVIlle siècle. Ils comprennent notamment le nom de chaque propriétaire, le nombre et la nature des bâtiments, ainsi que les superficies concédées et exploitées. L'étude du contexte de production, associé à la confection d'un papier terrier, révèle que ces aveux et dénombrements font partie intégrante de documents ayant une valeur légale et sanctionnant un droit de propriété. L’analyse a confirmé que les aveux couvraient adéquatement l'ensemble de la vallée laurentienne. Elle a, par ailleurs, fait ressortir un décalage chronologique occasionnel entre la date de confection de l'aveu et dénombrement et celle de son dépôt officiel. Il appert que la très grande majorité des aveux et dénombrements concernent la période 1723-1725. Ces documents se prêtent donc à une analyse détaillée de la spatialisation du peuplement au XVIII ${ }^{\mathrm{e}}$ siècle.
Tous droits réservés Institut d'histoire de l'Amérique française, 1989
Ce document est protégé par la loi sur le droit d'auteur. L’utilisation des services d'Érudit (y compris la reproduction) est assujettie à sa politique d'utilisation que vous pouvez consulter en ligne.

https://apropos.erudit.org/fr/usagers/politique-dutilisation/ 


\title{
LES AVEUX ET DÉNOMBREMENTS DU RÉGIME FRANÇAIS (1723-1745) ${ }^{1}$
}

\author{
JACQUES MATHIEU \\ ALAIN LABERGE \\ RENALD LESSARD \\ LINA GOUGER \\ CÉLAT, Université Laval
}

\section{RÉSUMÉ}

Ce texte présente une analyse critique de la série des aveux et dénombrements du Régime français dont nous voulions vérifier la validité, la représentativité et la fiabilité. Ces documents décrivent, terre par terre, l'implantation de la population dans la vallée laurentienne après le premier quart du XVIIIe siècle. Ils comprennent notamment le nom de chaque propriétaire, le nombre et la nature des bâtiments, ainsi que les superficies concédées et exploitées.

L'étude du contexte de production, associé à la confection d'un papier terrier, révèle que ces aveux et dénombrements font partie intégrante de documents ayant une valeur légale et sanctionnant un droit de propriété. L'analyse a confirmé que les aveux couvraient adéquatement l'ensemble de la vallée laurentienne. Elle a, par ailleurs, fait ressortir un décalage chronologique occasionnel entre la date de confection de l'aveu et dénombrement et celle de son dépôt officiel. Il appert que la très grande majorité des aveux et dénombrements concernent la période 1723-1725. Ces documents se prêtent donc à une analyse détaillée de la spatialisation du peuplement au XVIIIe siècle.

\section{ABSTRACT}

This paper presents a critical analysis of the aveux et dénombrements, a series of documents from the French regime, examining their validity, representativeness and reliability. They describe the landed property of the Laurentian valley after the first quarter of the eighteenth century, giving the name of each owner, a description of the buildings and the amount of land actually being exploited, as well as the size of the original concession.

The study of the context in which the aveux et dénombrements were produced shows that they represent a crucial part of a group of documents comprising the papier terrier which sanction the right of property and have legal value. The analysis confirms that the aveux et dénombrements cover most of the St.Lawrence valley quite adequately. The study does reveal certain discrepancies between the moment when the documents were drawn up and their official registration. Most of the aveux et dénombrements describe the period from 1723 to 1725 . Therefore they can indeed be used for a spatial analysis of settlement in the Laurentian valley at this time.

1 Cette recherche a reçu l'appui du CRSH et du fonds FCAR.

RHAF, vol.42, no 4, printemps 1989 
Les aveux et dénombrements constituent une source d'information tout à fait remarquable pour obtenir une connaissance détaillée de l'occupation de la vallée laurentienne dans la première moitié du XVIIIe siècle $^{2}$. Précisons cependant qu'ils n'ont rien de commun avec les recensements de la même époque, parfois également appelés dénombrements ${ }^{3}$. Ces recensements ne réunissent que des données agrégées, par paroisse ou par seigneurie. Les aveux et dénombrements réalisés entre 1723 et $1745^{4}$ en diffèrent par l'unité de base retenue. Ils décrivent chaque unité cadastrale de chaque seigneurie, fournissant ainsi une énumération et une description standardisées, terre par terre. Le relevé de chaque propriété comprend le nom du propriétaire, le nombre et la nature des bâtiments (maison, grange, étable, écurie, etc.) et, parfois, les dimensions et matériaux. Il mentionne la superficie de chaque censive concédée, en plus de celle des domaines, ainsi que la superfície exploitée. Il indique la nature de la mise en valeur par des précisions, comme en culture ou en prairies, et mentionne à l'occasion les superficies à la pioche, en abatis, etc. S'y ajoutent l'indication des cens, rentes et autres droits à payer, ainsi que la présence de moulins et de bâtiments de service.

Les aveux et dénombrements comportent plusieurs commentaires descriptifs ou qualitatifs sur la propriété seigneuriale, les équipements collectifs, les communes, les terres en voie de défrichement, etc. Mais ce sont les renseignements à caractère sériel qui présentent le plus d'intérêt. La description détaillée, terre par terre, pour l'ensemble de la vallée du Saint-Laurent se prête à de multiples études, comme la spatialisation du peuplement, certaines facettes des stratégies foncières familiales, les structures agraires ou les aménagements différenciés des censives. En somme, la série des aveux et dénombrements permet la constitution d'une véritable typologie des terres fondée sur une reconstitution au cas par cas et retenant en priorité l'influence de la localisation, de la qualité des sols, de la gestion seigneuriale et de la situation des familles.

Encore fallait-il vérifier au départ la validité, la représentativité et la fiabilité de cette source. L'étude du contexte de réalisation des aveux et dénombrements révèle une stratégie administrative et politique destinée à éviter les oppositions. Les réactions des seigneurs aux demandes des autorités coloniales et métropolitaines éclairent les rapports, assez

2 Jacques Mathieu et Réal Brisson, «La vallée laurentienne au XVIIIe siècle: un paysage à connaître», Cahiers de géographie du Québec, 28,73-74 (avril-sept 1984): 107-124.

3 Mario Boleda et Richard Laloue, «Une source en friche: les dénombrements sous le Régime français», Revue d' histoire de l'Amérique française, 42,1 (été 1988): 47-72.

4 Lors de la confection d'un papier terrier, commencé par Talon et continué par Duchesneau, un certain nombre d'aveux et dénombrements ont été produits en 1677 et 1678 . Une autre série a également été produite à la fin du XVIIIe siècle. Archives nationales du Québec à Québec (ANQ-Q), E21/38-39, ministère des Terres et Forêts, Aveux et dénombrements, régime anglais, 1777-1799. 
particuliers entre l'Église et l'État à propos de la gestion des terres, de même que tout l'aspect circonstanciel entourant la production de l'aveu et dénombrement et affectant la représentativité de cette source.

\section{LA NÉCESSITÉ D'UN PAPIER TERRIER}

Le contexte d'élaboration des aveux et dénombrements procède de deux grands motifs qui s'inscrivent dans la longue durée: la volonté d'accélérer la mise en valeur des terres et d'assurer le recouvrement des droits relevant du Domaine d'Occident. L'un et l'autre doivent s'appuyer sur un inventaire précis, complet et légal: un papier terrier, dont les aveux et dénombrements constituent une des parties essentielles.

La volonté de l'État d'obliger les seigneurs et les censitaires à mettre leurs terres en valeur remonte au XVIIe siècle. En 1663, dès l'instauration d'un gouvernement royal en Nouvelle-France, le Conseil d'État du roi ordonne de défricher les terres ${ }^{5}$. Par la suite, divers édits, arrêts et ordonnances issus tant de la métropole que de la colonie sont promulgués sur le même sujet. Ils aboutissent en 1711 aux fameux arrêts de Marly, une intervention royale qui se veut énergique et vise à la fois les seigneurs et les censitaires ${ }^{6}$. Estimant que les terres non défrichées retardent le développement du Canada, le roi fixe à un an le délai pour tenir feu et lieu et mettre les terres en valeur; à défaut de quoi les fautifs seront déchus de leurs titres de propriété. Les administrateurs coloniaux enregistrent rapidement ces arrêts, mais tardent à les mettre en application ${ }^{7}$. Malgré les rappels périodiques et pressants des autorités françaises, en 1719, rien de concret n'est encore fait, le gouverneur Vaudreuil et l'intendant Bégon prétextant que l'intention n'était que de faire planer une menace et de susciter la crainte ${ }^{8}$. L'étendue du Canada, l'absence de demandes de censives dans les lieux inexploités et le peu d'empressement des seigneurs à se déclarer sujets à une réunion de leur seigneurie au Domaine du roi auraient retardé l'application de cette réglementation. Du reste, la confection préalable d'un papier terrier identifiant toutes les seigneuries non encore établies leur paraît indispensable ${ }^{9}$.

Le recouvrement des droits relevant du Domaine d'Occident incite également à la confection d'un papier terrier. À partir de 1674, tous les droits perçus par la Compagnie des Indes occidentales sont transférés au Domaine d'Occident, lui-même un élément de la Ferme générale.

\footnotetext{
5 Arrêt du Conseil d'État du Roi, 21 mars 1663, publié dans Édits, ordonnances royaux, déclarations et arrêts du Conseil d'État du roi... (Québec, E. R. Fréchette, 1854), 1: 33.

6 Arrêt du Conseil d'État du Roi, 6 juillet 1711, Idem, tome 1: 326; R. C. Harris, The Seigneurial System in Early Canada (Québec, Presses de l'Université Laval, 1968), 106.

7 Ces arrêts sont insinués par le Conseil supérieur de Québec dès le 5 décembre 1712, de même que par la juridiction de Montréal et par la Prévôté de Québec le 31 janvier suivant.

8 Vaudreuil et Bégon au Conseil de marine, 26 octobre 1719, Archives des Colonies (AC), série C11A, vol. 40, f. 35v-36r.

9 Idem.
} 
En octobre 1719, François-Étienne Cugnet, qui vient d'arriver à Qué$\mathrm{bec}$, remplit les fonctions de directeur du Domaine d'Occident ${ }^{10}$. Il assume ainsi la responsabilité de la perception des droits de quint et de relief provenant de chaque mutation des propriétaires d'une seigneurie ${ }^{11}$ et pour cela un papier terrier s'impose. Il ne dispose alors que d'un ancien terrier du Domaine du roi confectionné à l'époque de l'intendant Duchesneau qui ne peut d'ailleurs «Servir attendu le grand nombre de terres concédées depuis qui n'Y (sont)... point comprises, et que celles qui y (sont)... portées ont changé de propriétaires et de confins par les nouveaux Etablissements»12. Bref, par ce document désuet confectionné 40 ans plus tôt, il est «impossible d'y rien connoitre» ${ }^{13}$. Dans ces circonstances, faute d'être connus ou de se faire connaître, plusieurs propriétaires peuvent devoir des sommes au Domaine sans que les administrateurs de celui-ci le sachent. De fait, entre 1723 et 1733 le montant des droits atteint une moyenne annuelle de 1100 livres dont 3979 livres pour la seule année 1730. Cugnet a tout intérêt à entrer dans les vues de l'intendant Bégon qui désire la confection d'un papier terrier.

Bégon met rapidement au point un plan d'action. Il écrit au contrôleur général, le marquis d'Argenson ${ }^{14}$, insistant sur la nécessité d'un terrier pour assurer le recouvrement des droits seigneuriaux. Il charge Cugnet d'en faire autant auprès des directeurs de la Ferme en s'engageant à en payer les coûts de réalisation à même l'État du Roi ${ }^{15}$. Dans la même foulée, en 1721, Cugnet se plaint de la désuétude des anciens terriers et il signale qu'il n'a reçu aucun paiement de droits pour l'année en cours. Bégon informe le Ministre des dispositions prises: contrôle des concessions et recensement des seigneuries, en vue de la réunion éventuelle des seigneuries inexploitées ${ }^{16}$. Finalement, l'intendant peut agréer à la requête de Cugnet faisant suite à l'ordre des directeurs de la

10 Cameron Nish, François-Étienne Cugnet 1719-1751: Entrepreneur et entreprises en Nouvelle-France (Montréal, Fides, 1975), 7, 12-14.

11 L'on retrouve également parmi ces droits les lods et ventes et les cens et rentes des censives relevant directement du roi. Mémoire de Hocquart sur la Régie du Domaine d'Occident en Canada, 1733, AC, série C11A, vol. 59, f. 333v-336r.

${ }_{12}$ Idem; ces propos, repris en 1733, sont identiques à ceux tenus par Cugnet dans une lettre datée du 4 novembre 1721 . Voir à ce sujet, Requête des fermiers généraux, 2 mai 1722, AC, série C11A, vol. 44, f. 408 r.

13 Idem.

14 Par ses fonctions, d'Argenson était chargé, entre autres, de la direction générale de toutes les fermes du roi et, par conséquent, du Domaine d'Occident.

15 L'intendant s'appuie constamment sur l'article 383 du bail consenti à Pierre Domerque le 18 mars 1687 qui lui fournit les assises légales pour justifier la confection d'un papier terrier.

16 Vaudreuil et Bégon au Conseil de marine, 26 octobre 1719, AC, série C11 A, vol. 40, f.36r-37r; Requête des fermiers généraux, 2 mai 1722, AC, série C11A, vol. 44, f. 408 r; Vaudręuil et Bégon au Conseil de marine, 17 octobre 1722, AC, série C11A, vol. 44, f. 295r-295v; Mémoire de Hocquart sur la Régie du Domaine d'Occident en Canada, 1733, AC, série C11A, vol. 59, f. 333v-336r. 
Ferme afin de procéder à la confection d'un papier terrier. Le 24 décembre 1722, il émet une ordonnance en ce sens ${ }^{17}$.

\section{LES ENJEUX DE LA CONFECTION D'UN PAPIER TERRIER}

Les raisons invoquées dans le préambule de l'ordonnance de Bégon passent sous silence la volonté de veiller à la mise en valeur des terres pour faire valoir, comme seul motif, la demande des Fermiers généraux $^{18}$. Tout indique que le prétexte occultait l'intention véritable. Dans leur correspondance avec le Ministre, les intendants font constamment référence à l'application des arrêts de Marly. La confection du papier terrier permettra de légaliser les titres de propriété, de contrôler le défrichement, voire de mettre de l'ordre dans les «papiers de l'Etat».

Les fondements juridiques d'une telle entreprise sont manifestes. Le papier terrier comporte quatre séries de documents dont la valeur légale est encore reconnue. Ce sont les copies des titres de propriété ${ }^{19}$, les actes de foi et hommage ${ }^{20}$, les aveux et dénombrements des seigneuries $^{21}$, de même que les déclarations des censitaires du roi ${ }^{22}$. Le terrier s'étend à tous les «fiefs relevans directement de Sa Majesté (et) de leurs dépendances», ainsi qu'aux "maisons, terres et héritages en roture estans en la Censive du roi». L'information que doivent contenir les aveux est également très précise:

bornes de chaque seigneurie, leur front, leur profondeur et leur situation au Nord et au Sud du fleuve $\mathbf{S}^{t}$ Laurent ou des Rivieres qui s'y dechargent L'etendue du Domaine des proprietaires des seigneuries Le nombre et la qualité de leurs batiments et moulins, et le nombre d'arpens (en) terres labourables et de prairies qui sont sur led. domaine Les tenants et aboutissants des habitations de leurs tenanciers Le nombre d'arpens de front et de profondeur qu'elles contiennent Les cens et rentes que chaque habitant paye

17 Ordonnance de l'intendant Michel Bégon, 24 décembre 1722, ANQ-Q, E 1/6, Ordonnances des intendants, Cahier 8, f. 139v-140v. terrier.

${ }_{18}$ Ce recours permettait sans doute de donner des bases juridiques plus solides au papier

19 Ces copies, signées par les seigneurs et contresignées par l'intendant, forment les Cahiers d'intendance. Ces documents, qui débutent en 1723 et se terminent en février 1725, comprennent deux volumes relatifs aux fiefs et seigneuries et deux autres concernant les censives du roi. ANQQ, E21/3-6, ministère des Terres et Forêts, Cahiers d'intendance.

${ }_{20}$ Par l'acte de foi et hommage, vestige du système féodal, un vassal se reconnaît dépendant d'un suzerain. Ce document comprend une description sommaire du fief et des titres de propriétés en plus d'un relevé des droits et devoirs du titulaire. ANQ-Q, E21/12-13, ministère des Terres et Forêts, Fois et hommages; voir aussi François-Joseph Cugnet, Traité de la Loi des Fiefs (Québec, Guillaume Brown, 1775), 1-6.

21 ANQ-Q, E21/17-20, ministère des Terres et Forêts, Aveux et dénombrements, Régime français.

${ }_{22}$ Les propriétaires de censives relevant directement du roi doivent faire une déclaration dans laquelle l'on retrouve une description précise à la fois des lieux et des titres de propriétés. Ces propriétés sont localisées principalement dans les villes de Québec et de Trois-Rivières mais également, pour un certain nombre de cas, dans les régions rurales. Ces documents ont été transcrits dans les registres contenant les aveux et dénombrements. 
par an pour chaque arpent de front, ou autres droits auxquels ils sont sujets Le nombre d'arpens de terre labourable et de prairies, et le nombre et qualité des bâtiments qui sont sur chaque habitation. 23

Ainsi les documents produits ont pour objet «non seulement la reconnoissance du seigneur Direct mais encore la conservation de L Intégrité de son fief ${ }^{24}$. Lorsque le suzerain reçoit l'aveu et dénombrement, il dispose de 40 jours pour l'accepter ou pour en refuser certains articles $^{25}$. Signés par les seigneurs et contresignés par l'intendant, ces documents prennent une valeur légale et confirment des devoirs autant que des droits.

Dans ces conditions, les procédures de vérification sont menées de manière rigoureuse. La responsabilité en est confiée au procureur du roi. Aidé de commis et en collaboration avec le directeur du Domaine d'Occident, il doit vérifier les titres de propriété, rechercher les informations manquantes et exécuter les copies. Il s'agit d'un travail de longue haleine, «plein de discussions», qui exige parfois, comme à Montréal, de passer d'habitation en habitation, ou encore, d'avoir recours aux actes notariés ${ }^{26}$. En tout, écrit le procureur Louis-Guillaume Verrier, «si je ne me prestois pas à tout ce détail... il seroit comme impossible de leur faire faire des déclarations aussi fidèles et en aussi bonne forme que les intérêts de Sa Majesté l'exigent» ${ }^{27}$. En somme, la confection d'un papier terrier sanctionne des droits légaux sur la propriété foncière et obéit à des procédures juridiques minutieuses.

La confection du papier terrier eut en outre des finalités administratives majeures. Constatant qu'il n'y avait pas de registre des anciennes

23 Vaudreuil et Bégon au ministre, 14 octobre 1723, AC, série C11A, vol. 45, f. 32v-34v; voir aussi Guyot, Répertoire universel et raisonné de jurisprudence civile, criminelle et bénéficiale (Paris, Panckoucke, 1776), tome 3: 503; le mode de compilation par les seigneurs des données relatives aux censitaires n'est pas bien établi. Dans un seul document, une ordonnance de 1732 forçant le Séminaire de Québec à remettre ses aveux et dénombrements, il est clairement indiqué que les tenanciers doivent se présenter avec leurs titres devant leur seigneur pour le mettre en état de compléter son aveu (Ordonnance de Gilles Hocquart, 21 mai 1732, Archives du Séminaire de Québec, Séminaire 40, no 26). Cette façon de procéder, qui est celle utilisée lors de la confection d'un terrier seigneurial, a-t-elle été adoptée uniformément de 1723 à 1745 ? Nous ne sommes pas en mesure de l'affirmer. Il n'est pas impossible de penser, en effet, que certains seigneurs, en particulier pour les petites seigneuries plus ou moins développées, aient pu recueillir les renseignements nécessaires de manière moins élaborée.

24 Le Conseil de marine à Vaudreuil, 22 mai 1724, AC, série B, 47: 1096.

25 En 1781, le gouverneur Haldimand refuse de signer les aveux du seigneur Chartier de Lotbinière sous prétexte qu'il ne reconnaît pas certaines portions de terre comme faisant partie de ses domaines. Le seigneur se voit ainsi contraint à modifier ses aveux et à les rendre conformes à ceux fournis en 1725. Chartier de Lotbinière à Haldimand, 17 juillet 1782, British Library, Add. MSS 21734, f. 503r-504v. $57 \mathrm{v}-60 \mathrm{r}$.

26 Beauharnois et Hocquart au ministre, ler octobre 1733, AC, série $\mathrm{C} 11 \mathrm{~A}$, vol. 59, f.

27 Louis-Guillaume Verrier, procureur général, au ministre, 9 octobre 1733, AC, série C11A, vol. 60 , f. $353 \mathrm{v}$. 
concessions, que seulement un petit nombre d'entre elles avaient été enregistrées au Conseil supérieur et que la plupart des confirmations par le Conseil d'État du roi ne contenaient que les noms des concessionnaires et la date de la concession sans en expliquer l'étendue et la situation, l'intendant Bégon fit copier les titres de propriété qui lui furent présentés. Pour plus de sûreté, il fit confectionner deux registres de copies ${ }^{28}$. Dans l'esprit de l'intendant Gilles Hocquart, ce terrier «sera pour toujours le fondement de Ceux qui pourront être faits dans la Suite et Servira longtemps en y ajoutant les nouvelles concessions qui seront accordées, et des mutations a mesure qu'elles arriveront ${ }^{29}$. Il envisageait même d'obliger les notaires et les greffiers à dresser tous les trois mois un état certifié des ventes, échanges et adjudications.

Tout au long de cette opération, les intendants ont cherché à établir avec précision l'état de la colonie et à dresser un papier terrier utile à des fins administratives et juridiques.

\section{LA RÉPONSE DES SEIGNEURS}

L'ordonnance de Bégon entraîne des réponses différentes de la part des seigneurs ${ }^{30}$. La majorité d'entre eux s'y conforment assez strictement dans des délais raisonnables. Mais certains ne donnent pas suite aux ordonnances, causant ainsi des retards à la clôture du papier terrier, tandis que les seigneurs ecclésiastiques s'y opposent carrément. L'analyse de la réaction des seigneurs aux ordonnances de confection d'un papier terrier éclaire la nature et les motifs des retards, en plus de préciser les qualités et valeurs représentatives des aveux et dénombrements aux fins d'un usage sériel.

La réponse des seigneurs se découpe en trois temps bien distincts: une première période très forte (1723-1726), suivie d'un creux important (1727-1730) et d'une reprise plutôt saccadée (1731-1745). La première période correspond aux dernières années de l'intendance de Bégon. Cent trente-cinq aveux et dénombrements sont alors reçus et enregistrés, mais selon un rythme irrégulier qui incite l'intendant à recourir à des ordonnances supplémentaires les 24 mai 1724 et 14 janvier $1725^{31}$. Jusqu'à son départ du Canada, l'intendant demeure optimiste quant à la conclusion prochaine du papier terrier.

\footnotetext{
${ }^{28}$ Hocquart au ministre, 17 octobre $1730, \mathrm{AC}$, série C11A, vol. 53, f. 84v.

29 Mémoire de Hocquart sur la Régie du Domaine d'Occident au Canada (1733), AC, série C11A, vol. 59 , f. 333r-336r.

30 L'analyse qui suit tient compte de l'ensemble des aveux et dénombrements à l'exception des fiefs exclusivement urbains.

${ }_{31}$ ANQ-Q, E1/7, Ordonnances des intendants, Bégon, 24 mai 1724, vol. 10, f. 28 r et 14 janvier 1725, vol. 11, f. 2 r. L'ordonnance de 1724 se distingue de toutes les autres par la présence d'un passage sur le «refus tacite» des retardataires «de rendre à Sa Majesté les devoirs par eux dus», «refus d'autant plus blâmable que lesd. propriétaires ont eu les seigneuries /.../ qu'ils possèdent à titre gratuit et de la pure libéralité de Sa Majesté».
} 


\section{Tableau $^{32}$}

\section{Répartition annuelle des enregistrements d'aveux et dénombrements}

$\begin{array}{cc}\text { Année } & \text { Nombre } \\ 1723 & 64 \\ 1724 & 32 \\ 1725 & 37 \\ 1726 & 2 \\ 1727 & \\ 1728 & 0 \\ 1729 & 0 \\ 1730 & 0 \\ & 0\end{array}$

$\begin{array}{rr}1731 & 3 \\ 1732 & 6 \\ 1733 & 11 \\ 1734 & 1 \\ 1735 & 0 \\ 1736 & 13 \\ 1737 & 3 \\ 1738 & 5 \\ 1739 & 3 \\ 1740 & 0 \\ 1741 & 0 \\ 1742 & 0 \\ 1743 & 1 \\ 1744 & 0 \\ 1745 & 9\end{array}$

Total

Malgré l'insistance grandissante de la Cour, une nouvelle ordonnance de l'intendant Dupuy en 1726 et une autre de Hocquart en $1729^{33}$, aucun aveu et dénombrement n'est enregistré jusqu'en 1731. Cette période est marquée par le décès du procureur général Collet en mars 1727. Le procureur jouait un rôle essentiel dans la confection du terrier

32 On dénombre 190 aveux et dénombrements qui se répartissent en 159 déclarations. Cette différence s'explique par la teneur de certaines déclarations. En effet, si ordinairement une déclaration contient l'aveu et dénombrement d'une seigneurie, il arrive aussi que le seigneur propriétaire de plusieurs fiefs procède à une seule déclaration contenant les aveux et dénombrements de chacune de ses seigneuries. Un aveu et dénombrement correspond le plus souvent à une seigneurie. Cependant, en raison de mutations de la propriété seigneuriale, il existe des aveux couvrant seulement une partie du territoire d'une seigneurie.

33 ANQ-Q, E1/8, Ordonnances des intendants, Dupuy, 24 décembre 1726, vol.12A, f. $14 \mathrm{v}$; Hocquart, 22 novembre 1729 , vol.17, f. 17r-18r. 
et, à sa mort, de nombreux documents et titres déposés chez lui par plusieurs seigneurs y étaient restés "pêle-mêle» ${ }^{34}$. L'intendant ClaudeThomas Dupuy entend remettre de l'ordre dans tout cela afin de pouvoir continuer le papier terrier, mais sa destitution accentue encore le vide administratif ${ }^{35}$. Ce n'est qu'en 1731 qu'une nouvelle équipe, constituée de l'intendant Gilles Hocquart et du procureur général Louis-Guillaume Verrier, reprend en main le dossier du papier terrier ${ }^{36}$.

La conclusion du papier terrier traîne tout de même en longueur: 55 aveux et dénombrements sont enregistrés entre 1731 et 1745 . Il était pourtant devenu impératif d'en finir car les promesses annuelles de Hocquart, ajoutées à toutes les autres depuis Bégon, finissent par agacer sérieusement le Ministre qui se dit «fâché» ${ }^{37}$. Il décide d'ailleurs de suspendre le versement des gratifications à Verrier jusqu'à la fin des travau $^{38}$. En 1736, devant le peu d'empressement des retardataires à se mettre en règle, l'intendant va jusqu'à donner une commission à l'un des préposés au papier terrier, le notaire Dulaurent, pour se rendre chez les seigneurs afin d'y recueillir leurs déclarations ${ }^{39}$. Ce geste extraordinaire indique bien à quelles extrémités en sont arrivées les autorités coloniales pour satisfaire la métropole. Enfin, le ler septembre 1740, le papier terrier est clos et «regardé comme complet par rapport aux anciennes concessions» ${ }^{40}$. Verrier projette déjà d'y ajouter des «suppléments» devant couvrir les nouvelles concessions «faites et à faire», ce qu'approuve sans réserve le Ministre ${ }^{41}$. Cependant, cette initiative reste lettre morte; à une exception près, les suppléments de 1743 et de 1746 contiennent des documents concernant des seigneuries anciennes qui n'étaient pas prêts lors de la clôture de 1740 . Ce sont là les derniers aveux et dénombrements du Régime français ${ }^{42}$.

Chez les seigneurs laïques, le retard à produire l'aveu s'explique par toutes sortes de circonstances particulières qui ne découlent pas de la mauvaise volonté. Il s'agit le plus souvent de problèmes de titres liés

\footnotetext{
34 Dupuy au Ministre, 20 octobre 1727, AC, C11A, vol.49, f. 320r-321r.

35 Cette destitution intervient à la suite d'un affrontement entre Dupuy et le gouverneur Beauharnois au lendemain de la mort de Monseigneur de Saint-Vallier. Voir Jean-Claude Dubé, Claude-Thomas Dupuy, intendant de la Nouvelle-France, 1678-1738 (Montréal, Fides, [1969]), 225-252.

36 Président du Conseil de Marine à Hocquart, 10 avril 1731, AC, série B, vol.55, f. 481v$482 r$.

7 Maurepas à Beauharnois et Hocquart, 24 mars 1733, AC, série B, vol.59, f. 408r.

38 Maurepas à Verrier, 15 mai 1736, AC, série B, vol.64, f. 449v.

39 ANQ-Q, E1/13, Ordonnances des intendants, Hocquart, 10 janvier 1736, vol.24, f. 1r-v.

40 Hocquart au Ministre, 4 octobre 1740, AC, série C11A, vol.73, f. 143v.

41 Verrier au Ministre, 14 octobre 1739, AC, C11A, vol. 72, f. 230v-232v; Maurepas à Verrier, 17 mai 1741, AC, série B, vol.72, f. 398v.

42 Il faut noter que les fois et hommages et les déclarations des censitaires du roi se poursuivent dans les années 1750 .
} 
à des partages successoraux ${ }^{43}$. L'attitude des seigneurs ecclésiastiques est plus complexe. Dès 1723, Bégon transmet au Ministre les prétentions des ecclésiastiques de «n'estre point tenus de fournir leurs aveux et dénombrements fondées sur les lettres d'amortissement qui leur ont esté accordées» ${ }^{44}$. De fait, un seul aveu et dénombrement ecclésiastique a été enregistré durant la première période (1723-1726) et les communautés constituent une bonne partie des retardataires.

Les communautés ont d'abord vérifié si elles étaient tenues de se plier aux exigences de l'État. Le clergé de France, en effet, était dispensé de rendre foi et hommage et de fournir aveu et dénombrement. C'est en tout cas la réflexion à laquelle se livre le procureur des Sulpiciens à Paris quand il écrit à ses collaborateurs montréalais en 1724 . Toutefois, il en vient rapidement à la conclusion qu'il faudra s'y soumettre puisqu'il s'agit du commencement du règne du Roi ${ }^{45}$. Il ajoute surtout que cela donne une excellente occasion d'obtenir de cette manière «un titre général et authentique de tous [nos] droits dans la suite». Il suggère de demander à l'intendant un délai «à l'amiable» pour permettre de travailler à la confection d'un nouveau terrier seigneurial de Montréal qui servira ensuite de base à l'aveu et dénombrement. Les lettres à terrier nécessaires sont envoyées de Paris de même qu'une procuration pour rendre foi et hommage ${ }^{46}$.

Si les seigneurs de Montréal décident assez rapidement de produire leurs aveux et dénombrements, tout porte à croire que d'autres seigneurs ecclésiastiques ont suivi le même exemple. Déjà, en mars 1725 ,

${ }^{43}$ C'est le cas de la seigneurie de Saint-Ours. Le seigneur Pierre de Saint-Ours rend foi et hommage en 1723 mais décède peu après sans avoir pu déposer l'aveu et dénombrement de son fief. Celui-ci ne sera remis qu'en 1745 et en différents fragments selon les héritiers. ANQ-Q, E21/ 12, ministère des Terres et Forêts, Foi et hommage, Régime français, 12 mai 1723, cahier 2, f. 66; Pierre-Georges Roy, Inventaire des concessions en fief et seigneurie, fois et hommages et aveux et dénombrements conservés aux Archives de la Province de Québec (Beauceville, L'Eclaireur, 1927), II: 171-174; ANQ-Q, E21/19, ministère des Terres et Forêts, Aveux et dénombrements, Régime français, vol.3, f. 108-150. La seigneurie de Saint-Augustin représente une facette extrême de ce problème: suite à l'endettement, à la faillite et au décès du seigneur en 1725 , ce fief est finalement confisqué et adjugé à ses créanciers six ans plus tard; Lucien Campeau, "Aubert de La Chesnaye, François», Dictionnaire biographique du Canada, II: De 1701 à 1740, 36-37; P.-G. Roy, Inventaire des concessions en fief et seigneurie..., I: 274. La possibilité de simple négligence de certains seigneurs n'est pas à exclure. François Chorel, seigneur de la moitié de Sainte-Anne-de-la-Pérade, rend foi et hommage en 1723 et ne dépose son aveu qu'en 1738 . ANQ$\mathrm{Q}, \mathrm{E} 21 / 12$, ministère des Terres et Forêts, Foi et hommage, Régime français, 3 février 1723, vol.2, f. 12; P.-G. Roy, ICFS, II: 153; ANQ-Q, E21/18, ministère des Terres et Forêts, Aveux et dénombrements, Régime français, vol.2, f. 634. Parfois aussi, l'apparence de négligence seigneuriale cache une tout autre réalité: la confusion de la bureaucratie coloniale. L'aveu et dénombrement de la seigneurie de Champlain n'est enregistré qu'en 1738, mais ce retard tient à ce que les papiers relatifs à ce fief sont restés ignorés plus de dix ans à l'intendance. Beauharnois et Hocquart au Ministre, 17 octobre 1733, AC, série C11A, vol. 59, f. 241r-242v.

44 Le Conseil de marine à Vaudreuil, 22 mai 1724, AC, série B, vol.47, f. 1091-1096; on y fait référence à une lettre de Bégon du 26 octobre 1723 relevant ce fait.

45 Selon le droit féodal, un nouveau suzerain peut en effet demander à ses vassaux de prêter foi et hommage et de fournir aveu et dénombrement.

${ }_{46}$ Père Pierre-François Magnien au père François de Chaumaux, 22 mai 1724, Archives nationales du Canada (ANC), MG 17, A 7-2, série II, vol.1, f. 731. 
les dames de l'Hôpital-Général de Québec ont déposé leur aveu et dénombrement pour leur moitié de la seigneurie de la Durantaye ${ }^{47}$. Aussi, en 1726, il est dit que les Jésuites ont «donné» leurs aveux et dénombrements ${ }^{48}$. Par contre, ces derniers s'opposent farouchement à rendre foi et hommage. Ils se montrent intraitables à ce chapitre et s'y refusent toujours, allant même jusqu'à en exposer les raisons dans le préambule de leurs aveux. Enfin, le Séminaire de Québec semble lui aussi disposé à se rendre aux désirs des autorités puisque ses titres ont été retrouvés chez Collet en $1727^{49}$.

En fait, les dates tardives d'enregistrement des aveux et dénombrements des Sulpiciens (1731) et des Jésuites (1733) découlent probablement davantage de la désorganisation administrative de la colonie entre 1727 et 1731 que d'une opposition soutenue. Le cas du Séminaire de Québec n'est pas aussi net. Les aveux et dénombrements de Beaupré et de l' ̂̂le-Jésus sont bien enregistrés en 1732, mais à la suite d'une ordonnance particulière ${ }^{50}$; et les autres aveux du Séminaire ne sont enregistrés qu'en 1738, reflétant peut-être plus qu'une certaine tiédeur envers l'obligation de fournir ces documents.

Les résistances furent plus grandes du côté des communautés féminines et en particulier chez les dames de l'Hôtel-Dieu de Québec dont il est souvent question dans la correspondance. Dès 1726, il est question de les obliger à se conformer aux ordonnances ${ }^{51}$. D'après le procureur Verrier toutefois, le problème de l'Hôtel-Dieu vient de la confusion de la gestion de ses seigneuries ${ }^{52}$, une lacune qui, selon lui, peut sans doute s'appliquer aux autres communautés de femmes. Le fait que tous leurs aveux et dénombrements soient enregistrés, sauf un, après 1735, va dans ce sens.

Dans l'ensemble, la collaboration des seigneurs à la constitution d'un papier terrier ne souffre pas de lacunes importantes. Comme les Sulpiciens, la plupart des seigneurs semblent avoir perçu l'intérêt de produire les aveux et dénombrements: ce type de document, tout en clarifiant la gestion de leurs fiefs, constituait, une fois accepté par l'État, une reconnaissance formelle de leurs droits et de leurs possessions. Ils y apportèrent donc beaucoup de sérieux. Ruette d'Auteuil, par exem-

\footnotetext{
47 ANQ-Q, E21/18, ministère des Terres et Forêts, Aveux et dénombrements, Régime français, vol. 1: «Fief de la Durantaye», 17 mars 1725, f. 216v.

48 Ministre à Dupuy, 14 mai 1726, $\mathrm{AC}$, série $\mathrm{B}$, vol.49, f. 672.

49 Hocquart au Ministre, 25 octobre 1729, AC, série C11A, vol. 51, f. 298v-299r

50 ANQ-Q, E1/11, Ordonnances des intendants, Hocquart, 21 mai 1732, vol. 20, f. 65r.

51 Ministre à Dupuy, 14 mai 1726, $\mathrm{AC}$, série $\mathrm{B}$, vol.49, f. 672.

52 Il déclare à ce propos: «Elle (cette communauté) manque pour le temporel, d'économe, agent ou homme d'affaires qui soit au fait et qui puisse à propos instruire d'une infinité de particularités nécessaires sur la nature et consistance des terres et domaines qui lui appartiennent et inconnues aux religieuses qui ne sortent point de leur maison.» Verrier au Ministre, 15 octobre 1738, AC, série C11A, vol.70, f. 202r-202v.
} 
ple, tient à corriger les dimensions erronées de sa seigneurie de la Pocatière contenues dans l'aveu et dénombrement que son fils avait fait enregistrer deux ans auparavant lors de son absence du Canada ${ }^{53}$. De même, Jean-François Bélanger procède, en 1739, à un nouvel aveu de la seigneurie de Bonsecours pour laquelle son père en avait pourtant déposé un dès $1723^{54}$. Certains seigneurs vont même jusqu'à produire des aveux et dénombrements pour des seigneuries éloignées servant à la pêche en Gaspésie ou sur la Côte-Nord; d'autres s'empressent d'en fournir pour des concessions très récentes non ratifiées ou encore au statut carrément équivoque; il existe même un aveu et dénombrement pour une seigneurie située en Acadie. On observe une tendance similaire du côté des seigneurs ecclésiastiques. Chez les Sulpiciens, le procureur analyse en profondeur la procédure à suivre et recommande «de ne rien omettre» au nouveau terrier ${ }^{55}$. Autre indice de la valeur du travail accompli lors de la confection des aveux et dénombrements: les copies seigneuriales des aveux des Jésuites pour Sillery et Prairie-dela-Madeleine ont vraisemblablement servi de documents de base à la préparation des papiers terriers de ces deux seigneuries en 1753 et 1761 . Enfin, les religieuses de l'Hôtel-Dieu de Québec, constatant le désordre de la gestion de la seigneurie de Saint-Augustin qui leur a été adjugée en 1734, retiennent les services du notaire Charles-Hilarion Dulaurent afin de procéder à la confection d'un terrier seigneurial et de l'aveu et dénombrement de ce fief.

En définitive, c'est un constat de bonne volonté doublée d'une intention manifeste de pouvoir profiter d'un acquis légal qui ressort de cet examen de la réponse seigneuriale.

\section{REPRÉSENTATIVITÉ DES AVEUX ET DÉNOMBREMENTS}

Les aveux et dénombrements seigneuriaux constituent un ensemble imposant: 190 documents dont 135 enregistrés entre 1723 et 1726 et 55 autres enregistrés entre 1731 et 1745 . Leur représentativité doit cependant être examinée sous deux angles: celui de la couverture spatiale et celui de l'étalement chronologique.

\section{La couverture spatiale}

Au niveau de la représentation spatiale, seule une poignée de toutes les seigneuries concédées avant 1723 demeurent sans aveu et dénom-

53 ANQ-Q, E21/17, ministère des Terres et Forêts, Aveux et dénombrements, Régime français, 5 mars 1723 et 12 mai 1725, vol.1, f. 34 et 237 .

54 ANQ-Q, E21/17, ministère des Terres et Forêts, Aveux et dénombrements, Régime français, vol. 1, «fief de Bonsecour», 26 février 1723, fol. 30 et E21/18, vol. 2, «fief de Bonsecour» 23 mars 1739 , f. $642 \mathrm{v}$.

55 Père Pierre-François Magnien au père François de Chaumaux, 22 mai 1724, ANC, MG 17, A 7-2, série II, vol. 1, f. 731. 
brement: deux dans le gouvernement de Montréal, deux autres dans celui des Trois-Rivières et neuf dans le gouvernement de Québec - en prenant les Éboulements et Rimouski comme limites orientales ${ }^{56}$. Quatre de ces seigneuries sont situées en dehors de la zone principale d'occupation: Petite-Nation et Pointe-à-l'Orignal dans l'Outaouais, le Bic loin au nord-est dans le Bas Saint-Laurent et Lessard à l'intérieur des terres derrière l'Islet-Saint-Jean sur la Côte-du-Sud; ces territoires marginaux sont encore inoccupés. En fait, ils n'apparaissent même pas sur la liste des seigneuries de 1728 qui a probablement servi de document de contrôle pour le gouvernement ${ }^{57}$. Les neuf autres seigneuries, situées dans le corridor laurentien, sont toutes recensées en 1721 dans le rapport du procureur général Collet sur l'organisation paroissiale de la colonie ${ }^{58}$. Trois d'entre elles sont cependant exclues de la liste de 1728, ce qui expliquerait en partie l'absence d'aveux dans leur cas ${ }^{59}$. Dans les six autres cas, des problèmes de nature juridique, successorale ou administrative peuvent expliquer l'absence d'aveu et dénombrement. À l'évidence, une bien faible portion du territoire seigneurial a échappé à l'élaboration du terrier.

Au surplus, ces absences ne touchent que des superficies et une population limitées. La plus importante des seigneuries sans aveu et dénombrement, les Aulnaies, ne compte en 1721 que « 24 chefs de famille résidents et trois concessionnaires» répartis sur trois lieues de front. D'autres seigneuries, comme Duquet, Vitré et Lafrenaye couvrent à peine quelques arpents de front et comptent très peu d'habitants. Ensemble, ces neufs seigneuries font environ dix lieues de front et totalisent autour d'une centaine de familles dans les années $1720^{60}$. Elles représentent environ $1,5 \%$ des censives et de la population. Il s'avère donc que les aveux et dénombrements seigneuriaux couvrent pratiquement la totalité de la vallée du Saint-Laurent et qu'ils permettent une reconstitution détaillée du paysage rural québécois de cette époque

\footnotetext{
56 Ces seigneuries sont Petite-Nation et Pointe-à-l'Orignal dans le gouvernement de Montréal, Nicolet et Dutort dans celui des Trois-Rivières et Deschaillons, Duquet, Vitré, Vincennes, Lafrenaye, Lessard, Les Aulnaies, Saint-Denis et le Bic dans le gouvernement de Québec. La carte de référence des seigneuries est Louise Dechêne, «Les seigneuries», R. Cole Harris, dir., Atlas historique du Canada, I: Des origines à 1800 (Montréal, Presses de l'Université de Montréal, 1987), planche 51 .

57 Cette liste accompagne l'extrait du papier terrier. Voir Gilles Héon, Répertoire numérique des anciennes archives françaises conservées au Centre d'archives de Québec ([Québec], Archives nationales du Québec, [1986]), 27.

58 [Mathieu-Benoît Collet], «Procès-verbaux sur la commodité et l'incommodité dressés dans chacune des paroisses de la Nouvelle-France par M.-B. Collet, procureur du Roi au Conseil supérieur de Québec», Rapport de l'archiviste de la province de Québec (1921-22), 347.

59 Il s'agit de Vitré, Vincennes et Saint-Denis. À remarquer que ce sont de petits fiefs intercalés entre d'autres plus importants.

60 Cette estimation est basée sur le rapport de Mathieu-Benoît Collet de 1721, op. cit.
} 


\section{L'étalement chronologique des aveux}

L'étalement chronologique des aveux soulève de plus sérieuses réserves quant à leur représentativité globale. Des écarts de temps trop grands et trop fréquents entre les aveux risqueraient de mélanger des réalités différentes, compte tenu de la forte évolution démographique du Canada durant cette période. En fait, près des trois quarts des aveux et dénombrements $(135 / 190 ; 71,1 \%)$ sont enregistrés dans les années 1723-1726. De plus, les 55 aveux postérieurs peuvent être ramenés à une quarantaine, si l'on regroupe les aveux partiels (il y en a cinq pour la seigneurie de Saint-Ours) et si l'on exclut les aveux des fiefs inexploités. Enfin, il y a tout lieu de croire que plusieurs autres aveux des années 1730 reflètent la réalité des années 1725 . C'est dans le cours de la vérification de la fiabilité des aveux et, plus précisément, par la comparaison des différentes versions, que s'est posé le problème de la date de confection de ceux déposés après $1731^{61}$.

Il existe, pour ainsi dire, trois exemplaires de la série d'aveux et dénombrements réalisée entre 1723 et 1745 . La version officielle conservée par les autorités de la colonie ${ }^{62}$ et la copie envoyée en France ${ }^{63}$ comprennent exactement les mêmes déclarations dans le même ordre. Pour ce qui est des copies seigneuriales, elle ne sont souvent en fait qu'une copie secondaire de la version gouvernementale ${ }^{64}$ et n'apportent

61 Sylvie Dépatie a aussi noté que l'aveu de l'île Jésus daté du ler juin 1732 avait été dressé au plus tard en octobre 1730. Nous la remercions des renseignements supplémentaires qu'elle nous a fournis. Sylvie Dépatie, «La structure agraire à l'île Jésus dans la première moitié du XVIIIe siècle; résultats préliminaires et critique des sources», François Lebrun et Normand Séguin, dir., Sociétés villageoises et rapports villes-campagnes au Québec et dans la France de l'Ouest XVIIe-XXe siècles. Actes du colloque franco-québécois (Québec, 1985) (Trois-Rivières, Centre de recherches en études québécoises, Université du Québec à Trois-Rivières/Presses universitaires de Rennes 2, 1987), 25-38.

62 Il s'agit de la copie originale des aveux et dénombrements conservée aux Archives nationales du Québec. C'est cette copie signée par le seigneur et contresignée par l'intendant qui est utilisée comme document de référence.

63 La copie française est réunie en huit volumes correspondant aux registres expédiés par Verrier entre 1735 et 1746 et dont une version microfilmée est conservée aux Archives nationales du Canada: Archives des colonies, Série G1, vol. 450 à 453, Aveux et dénombrements 17231733; vol. 454, Supplément 1743; vol. 455 à 457, Aveux et dénombrements et déclarations 17361740 .

64 Nous avons répertorié des versions d'aveu et dénombrement tirées des cahiers gouvernementaux et conservées dans les papiers seigneuriaux pour les seigneuries suivantes: Rivière du Sud, ANQ-Q, P52/11, Couillard-Després, doc. 809, «Aveu et dénombrement de J. Bte Couillard pour le fief et seigneurie de la Rivière-du-Sud», 10 avril 1732; Beauport, ANQ-Q, P240/4, Fonds Seigneurie, «Aveu et dénombrement du fief de Beauport», 1er juin 1725 (copie dactylographiée); Lotbinière, ANQ-Q, Z1/4, Papier Joly de Lotbinière, «Aveu et dénombrement du fief et seigneurie de Lotbinière», 1724; Notre-Dame-des-Anges, St-Gabriel, Batiscan, Cap-de-la-Madeleine, Prairie-de-la-Madeleine, Sillery, Bélair, ANQ-Q, E21/67-68, ministère des Terres et Forêts, Biens des Jésuites; St-Maurice, Archives du Séminaire de Québec, Polygraphie 3, No 92, «Aveu et dénombrement du fief de St-Maurice donné par le Sr Pierre Poulin», 5 avril 1725; Châteauguay, ANC, MG 8, F 15, "Aveu et dénombrement du fief par Zacharie Robutel sieur de La Noue», 19 juin 1724; Vaudreuil, ANC, MG 8, F 93, fos 1-12, «Aveu et dénombrement rendu par Philippe de Rigaud de Vaudreuil», 2 rnars 1725 (copie dactylographiée). Par contre, la mention relevée par Louise Dechêne de la «déclaration pour le papier-terrier de 1731» de la seigneurie de Montréal, microfilmée par les Archives nationales du Canada à partir des archives des Sulpiciens, est erronée. Il s'agit en fait de documents de 1781. Louise Dechêne, Habitants et marchands de Montréal au XVIIe siècle (Paris, Plon, 1974), 550. 
alors rien de neuf. Par contre, nous avons retrouvé la majeure partie des originaux des aveux et dénombrements des seigneuries des Jésuites.

Entre le 17 janvier et le 20 avril 1733, les Jésuites déposent six déclarations contenant onze aveux et dénombrements pour l'ensemble de leurs seigneuries ${ }^{65}$. Les documents originaux de leurs déclarations ont été retrouvés pour les seigneuries de Notre-Dame-des-Anges, Sillery, Saint-Gabriel et Prairie-de-la-Madeleine ${ }^{66}$, tandis que d'autres documents concernaient les autres seigneuries. Les documents seigneuriaux et les copies gouvernementales officielles présentent la même description détaillée des censives. Par contre sur trois documents seigneuriaux, des corrections ont été apportées à l'identité des propriétaires ${ }^{67}$. C'est l'analyse de ces corrections qui a permis d'établir avec précision le moment de confection des aveux.

Les changements de nom faisaient suite à une transaction ou, plus souvent, à un décès. Dans la seigneurie de Notre-Dame-des-Anges, la correction la plus ancienne a trait à la vente d'une terre survenue en juillet $1723^{68}$. Les 18 autres changements postérieurs font remonter la correction la plus tardive à celle du décès d'Antoine Huppé Lagroise le 21 novembre 1731. La situation est similaire dans les seigneuries de Sillery et de Saint-Gabriel. Les 18 corrections ont trait à des changements de propriétaire survenus entre avril 1724 et avril $1732^{69}$.

Les Jésuites ont manifestement fait procéder à une certaine mise à jour de leurs aveux avant de les déposer officiellement. Cette mise à jour a été réalisée entre avril 1732 et les premiers mois de 1733. Si les noms des propriétaires ont été changés, aucune correction n'a été apportée à la description des censives, qu'il s'agisse des bâtiments ou des superficies des terres en culture. Ainsi, la copie officielle des aveux et dénombrements de ces seigneuries superpose une réalité foncière des années 1723-1724 à une liste des propriétaires en 1732-1733.

Pour les autres seigneuries des Jésuites, les documents seigneuriaux sont quelque peu différents. L'aveu de Prairie-de-la-Madeleine,

65 ANQ-Q, E21/18, ministère des Terres et Forêts, Aveux et dénombrements, Régime français, vol. 2: «Fiefs appartenant aux Jésuites dans le gouvernement de Québec», 17 janvier 1733, f. 383v-397v; «Fiefs des Jésuites dans le gouvernement de Trois-Rivières», 20 février 1733, f. 397v-403v; «Fief de la Prairie de la Madelaine», 4 mars 1733, f. 403v-411v; «Fief de Sillery», 15 avril 1733, f. 411v-415r; «Fief de Belair ou Montagne à Bonhomme», 20 avril 1733, f. 415r416r; «Fief du Sault St-Louis», 20 avril 1733, f. 416r-417r.

${ }_{66}$ Notre-Dame-des-Anges: ANQ-Q, E21/157, ministère des Terres et Forêts, Biens des Jésuites, "Aveu et Dénombrement de Notre-Dame-des-Anges»; Sillery et St-Gabriel: ANQ-Q, E21/189, ministère des Terres et Forêts, Biens des Jésuites, «Aveux et dénombrement de Sillery»; Prairie-de-la-Madeleine: ANQ-Q, E21/146, ministère des Terres et Forêts, Biens des Jésuites, «Aveu et dénombrement».

67 Des noms de propriétaires ont été rayés et remplacés par d'autres, inscrits dans une calligraphie différente. On note aussi parfois l'ajout de quelques terres au bout des rangs.

68 Il s'agit de la terre des sieurs Riverin vendue à Simon Réaume le 19 juillet 1723 .

69 Les corrections commencent le 3 avril 1724 par le décès de Jacques Fréchet et elles intègrent des changements jusqu'en 1732 avec le décès de Jean Kescanivet dit Lespérance survenu le 6 avril 1732 . 
aussi élaboré en 1723, n'a pas été mis à jour. Le moment de confection a été déduit de la mention de l'âge d'un orphelin. Le document signale «un orphelin agé de 2 ans, fils de feu René Loingtain et petit fils de feu Jérôme Loingtain» propriétaire de plusieurs terres de la seigneurie. En fait, il s'agit de Pierre Longtain, né le 14 mars 1721 à Laprairie. La copie gouvernementale contient les mêmes renseignements que le texte seigneurial à l'exception de précisions particulières comme les mentions de terres affermées et celles des lieux de résidence des propriétaires.

Aucune version seigneuriale d'aveu et dénombrement n'a pu être retrouvée pour la seigneurie de Batiscan, mais deux documents seigneuriaux ont servi à la comparaison. Le premier de ces documents dresse un état des cens et rentes que chaque propriétaire doit aux seigneurs en $1720^{70}$. Ce document est remis à jour plus tard car il comprend les changements de propriétaire et les concessions postérieures à 1723 . Mais ces informations ne sont pas intégrées dans la copie gouvernementale. Le second document, anonyme, non daté et sans titre, couvre les censives de Batiscan et celles d'un petit fief situé à Trois-Rivières ${ }^{71}$. Complémentaire au premier, il répond néanmoins à une demande de précision de la part des seigneurs. Ce document est postérieur à 1721 et antérieur à octobre 1723, comme l'indiquent les vérifications de décès ou non des propriétaires. Avec ces deux documents, les seigneurs de Batiscan avaient toutes les informations nécessaires à la confection de l'aveu et dénombrement de la seigneurie de Batiscan et l'essentiel de la déclaration du fief de Trois-Rivières.

La seigneurie du Cap-de-la-Madeleine est la seule seigneurie rurale d'importance appartenant aux Jésuites pour laquelle on n'a pu retrouver de document de comparaison dans les papiers seigneuriaux. Par contre, une vérification portant sur les décès de censitaires mentionnés dans l'aveu et dénombrement, indique que le document a été élaboré en 1723.

La comparaison des documents seigneuriaux avec les copies officielles des aveux et dénombrements gouvernementaux pour les six seigneuries des Jésuites révèle donc que même s'ils ont été déposés et enregistrés officiellement en 1733, ils décrivent une réalité foncière de 1723 . Dès lors, une vérification s'imposait pour toutes les autres seigneuries dont l'aveu est postérieur à 1731 .

70 ANQ-Q, E21/100, ministère des Terres et Forêts, Biens des Jésuites, dossier: Compte des rentes dues 1720, «Cahier des cens et rentes des terres de Batiscan établi en octobre 1720». Ce document mentionne le nom des propriétaires, la dimension des terres (uniquement le front pour les terres de la rivière, leur profondeur étant indiquée généralement par leurs bornes naturelles ou autres) et les cens et rentes à payer.

71 ANQ-Q, E21/100, ministère des Terres et Forêts, Biens des Jésuites, Dossier: «Papier terrier 1669-1821». Le contenu est présenté sous forme de tableau et contient les noms des propriétaires, les dimensions des terres, les espaces labourables et en prairies, les bâtiments, en se limitant aux maisons et aux granges, les terres sans bâtiment, en bois debout et abandonnées. 
Il a été impossible jusqu'à maintenant de retrouver d'autres documents seigneuriaux d'aveu et dénombrement. La vérification a donc porté sur l'identification des propriétaires en regard de la date de leur décès. Dans au moins trois cas, les seigneuries de l'Islet St-Jean, de Lespinay et de la Rivière-du-Sud ${ }^{72}$, les aveux et dénombrements ont été confectionnés plusieurs années avant leur dépôt au gouvernement. Dans d'autres cas, il y a concordance entre l'identité des propriétaires et la date de dépôt de l'aveu. Il demeure possible cependant que, à l'exemple de quelques seigneuries des Jésuites, les noms des propriétaires de terres aient été ajustés, mais pas la description des terres. Il faudra pour s'en assurer, avoir recours à d'autres documents de vérification.

Somme toute, cet examen de la fiabilité des aveux et dénombrements démontre qu'il ne faut pas confondre la date d'enregistrement avec celle du relevé seigneurial. Au moins neuf des aveux déposés après 1731, sur les 18 que nous avons pu vérifier et qui comptent parmi les plus importants, décrivent une réalité des années 1723-1724.

Une dizaine d'années après les arrêts de Marly, les autorités de la Nouvelle-France entreprennent une vaste opération de reconnaissance du domaine seigneurial dans la vallée du Saint-Laurent et de régularisation de sa gestion. L'étude du contexte d'élaboration de ce projet montre bien les motifs qui animent les intendants: perspectives de réunion des fiefs inexploités, intérêts financiers pour le Domaine d'Occident, mais, de plus en plus, constitution d'un instrument de contrôle légalement reconnu et administrativement utile aux fins de l'occupation et de la mise en valeur des terres.

L'entreprise quasi démesurée de confectionner un papier terrier du Canada sous le Régime français au début du XVIIIle siècle eut des effets variables selon les groupes concernés. Au niveau gouvernemental, les résultats qu'on attendait du papier terrier en général et des aveux et dénombrements en particulier ne se sont pas directement concrétisés. Les autorités de la colonie ne se sont pas servies de ces documents dans le but de réunir des seigneuries au Domaine du Roi puisque les aveux n'ont pas été mis à jour après leur enregistrement. Les réunions de seigneuries auxquelles se résout Hocquart en 1741 ne portèrent que sur des concessions récentes dans la région du lac Champlain et pour lesquelles il n'y a pas d'aveu et dénombrement ${ }^{73}$. Certaines des finalités des autorités métropolitaines et coloniales furent toutefois atteintes de façon indirecte. À compter de 1720, on dénombre quelques centaines de requêtes des seigneurs suivies d'une ordonnance de l'intendant por-

72 ANQ-Q, E21/17, ministère des Terres et Forêts, Aveux et dénombrements, Régime français, vol. 1, «fief de la Rivière du Sud», 10 avril 1732, f. 362v-367r; «fief de l'Islet St-Jean», 13 avril 1732, f. 367r-367v; «fief de Lespinay ci devant St-Joseph», 1er mai 1732, f. 367v.

73 R. C. Harris, op. cit., 113. 
tant sur les titres, les redevances et la mise en valeur des terres. Il s'agit là d'une évolution sensible des préoccupations dans ce domaine.

Du point de vue des droits du Domaine d'Occident, l'opération n'a pas eu les suites financières espérées. Un mémoire de 1733 signale tout simplement que le papier terrier sera utile une fois en règle ${ }^{74}$. Mais, jusqu'à cette date, les montants perçus n'ont pas subi d'augmentation significative. Les autorités semblent avoir persévéré dans cette voie au niveau des censives du roi puisque, en 1758, les Jésuites et l'HôtelDieu de Québec sont condamnés à payer des arrérages importants pour des terrains situés à Québec.

Apparemment les seigneurs semblent avoir pleinement profité de la réalisation d'un papier terrier comprenant la présentation d'aveux et dénombrements. Forcés par ordonnance de produire ces documents, les seigneurs en ont finalement profité pour obtenir une reconnaissance officielle de leurs possessions et des droits s'y rattachant. Un bon nombre d'entre eux ont ainsi procédé à une régularisation de leur propre gestion.

En définitive, les aveux et dénombrements couvrent la très grande majorité des seigneuries peuplées de la vallée laurentienne. Pour l'essentiel, ils décrivent, minutieusement, la situation des aménagements fonciers entre 1723 et 1725 . Ils livrent une représentation concrète et détaillée de la propriété foncière et des stratégies d'aménagement et d'établissement des seigneurs et des censitaires dans la vallée du SaintLaurent.

74 Mémoire de Hocquart sur la Régie du Domaine d'Occident au Canada (1733), AC, série C11A, vol. 59, f. 333r-336r. 